\title{
Accuracy of MRI diagnosis of early osteonecrosis of the femoral head: a meta-analysis and systematic review
}

\author{
Ya-Zhou Zhang ${ }^{\dagger}$, Xu-Yang Cao ${ }^{\dagger}$, Xi-Cheng Li ${ }^{*} \mathbb{D}$, Jia Chen, Yue-Yuan Zhao, Zhi Tian and Wang Zheng
}

\begin{abstract}
Objective: To evaluate the overall diagnostic value related to magnetic resonance imaging (MRI) in patients with early osteonecrosis of the femoral head.

Methods: By searching multiple databases and sources, including PubMed, Cochrane, and Embase database, by the index words updated in December 2017, qualified studies were identified and relevant literature sources were also searched. The qualified studies included prospective cohort studies and cross-sectional studies. Heterogeneity of the included studies were reviewed to select proper effect model for pooled weighted sensitivity, specificity, and diagnostic odds ratio (DOR). Summary receiver operating characteristic (SROC) analyses were performed for meniscal tears.

Results: Forty-three studies related to diagnostic accuracy of MRI to detect early osteonecrosis of the femoral head were involved in the meta-analysis. The global sensitivity and specificity of MRI in early osteonecrosis of the femoral head were $93.0 \%$ (95\% Cl 92.0-94.0\%) and 91.0\% (95\% Cl 89.0\%-93.0\%), respectively. The global positive likelihood ratio and global negative likelihood ratio of MRI in early osteonecrosis of the femoral head were $2.74(95 \% \mathrm{Cl} 1.98-3.79)$ and 0.18 (95\% Cl 0.14-0.23), respectively. The global DOR was 27.27 (95\% Cl 17.02-43.67), and the area under the SROC was 93.38\% (95\% Cl 90.87\%-95.89\%).

Conclusions: This review provides a systematic review and meta-analysis to evaluate the diagnostic accuracy of MRI in early osteonecrosis of the femoral head. Moderate to strong evidence indicated that MRI appears to be significantly associated with higher diagnostic accuracy for early osteonecrosis of the femoral head.
\end{abstract}

Keywords: Meta-analysis, Early osteonecrosis of the femoral head, Magnetic resonance imaging, Diagnostic accuracy

\section{Background}

Avascular Necrosis of Femur Head (ANFH), or osteonecrosis of the femoral head, is a pathologic process, which was first seen in the weight-bearing area of the femur. The stress can lead to bone trabecular structure injury (microfracture) and influence the repair process of the femur, and if not managed timely, it leads to the collapse and deformation of the femur. With many etiological factors, ANFH results from interruption of blood supply to the bone and then leads to ischemic necrosis. ANFH can be divided in

\footnotetext{
* Correspondence: zccsfh@163.com

${ }^{\dagger}$ Ya-Zhou Zhang and Xu-Yang Cao contributed equally to this work. Department of Orthopedics, Heibei General Hospital, No. 348 Heping East Road, Shijiazhuang 050051, Hebei, China
}

traumatic ANFH and non-traumatic ANFH with the non-traumatic ANFH further dividing into steroid-induced and alcoholic non-traumatic ANFH and so on. The timely treatment of early ANFH could promote the recovery the disease. However, in the late stage, it results in femur collapse, loss of hip function, and a very poor outcome that affects the quality of life. Therefore, the early diagnosis of ANFH is of great significance [1-3].

Several methods for early diagnosis of ANFH have been proposed, including MRI, SPECT, CT, X-ray, DSA, and laser Doppler with different characteristics. MRI has been characterized as being non-invasive, rapid and high sensitive, and commonly used by many clinicians [4-6]. Furthermore, MRI has been used in many studies in the

(C) The Author(s). 2018 Open Access This article is distributed under the terms of the Creative Commons Attribution 4.0 International License (http://creativecommons.org/licenses/by/4.0/), which permits unrestricted use, distribution, and 
2092 articles searched from databases using the index words

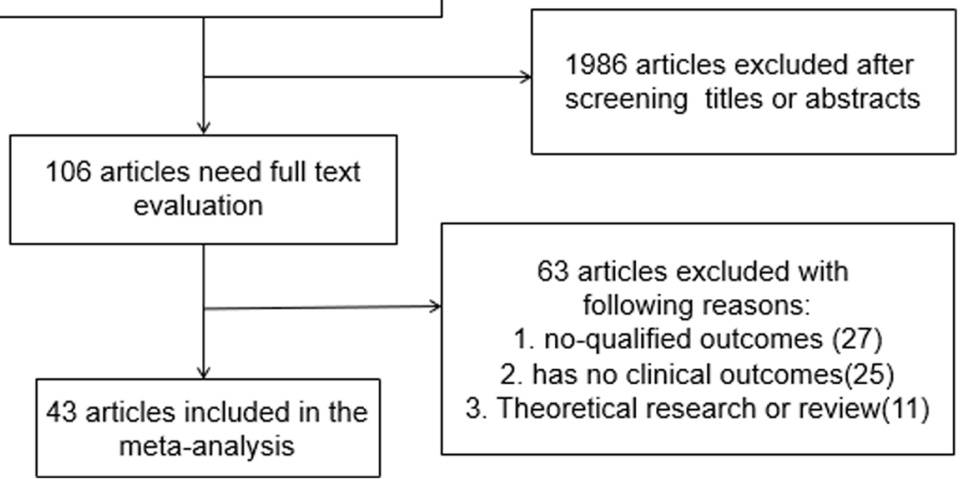

Fig. 1 Flow diagram of the literature search and selection process

diagnosis of early ANFH. Therefore, in this paper, a systematic review and meta-analysis of all qualified studies were performed to explore the diagnosis accuracy of MRI in early ANFH.

\section{Methods}

\section{Search strategy}

The following electronic databases were searched from their inception to December 2017: The Cochrane, PubMed, Embase database, for all the qualified trails that analyze the diagnostic accuracy of MRI of early osteonecrosis of the femoral head. Other related articles and reference materials were also identified for additional available studies. The literatures were searched independently by two investigators, and a third investigator was involved to reach an agreement.

\section{Study selection}

The studies that met the following criteria were included in our review: (1) prospective cohort study or cross-sectional study; (2) the research objects are patients suspected with early osteonecrosis of the femoral head without other serious diseases; (3) the studies provided the data of true positive (TP), false positive (FP), false negative (FN), and true negative (TN); and (4) the publications were only available in English and Chinese.

The studies that met the following criteria were excluded in our review: (1) repeat publications, or shared content and results; (2) case report, theoretical research, conference report, systematic review, meta-analysis, expert comment, and economic analysis; (3) the outcomes were not relevant; and (4) two or more results of the TP, FP, FN, and TN were zero.

\section{Data extraction and quality assessment}

Two independent investigators extracted the following data based on predefined criteria. Differences were settled by discussion with a third reviewer. The analyses data were extracted from all the included studies and consisted of two parts: basic information and main outcomes. The first part was about the basic information: the author name, the sample size, the percentage of male, and the age. The second part was the clinical outcomes. A $2 \times 2$ contingency table was constructed for each selected study; the results corresponding to the gold standard and MRI were selected as positive or negative. The data included true positive (TP), false positive (FP), false negative $(\mathrm{FN})$, and true negative $(\mathrm{TN})$. In studies in which one single cell in the $2 \times 2$ contingency table had a value of $0,0.5$ were added to all of the cells for calculation. Sensitivity, specificity, and likelihood ratio were calculated respectively, and the diagnostic odds ratio (DOR) was used as the measure of diagnostic accuracy. A DOR value of 1 indicates a test without discriminatory power, and the higher the DOR value is, the greater the degree of relevance of the assessed diagnostic test. The studies were performed by two reviewers independently. Any arising difference was resolved by discussion.

\section{Statistical analysis}

All statistical analyses were performed in the STATA 10.0 (TX, USA). Chi-squared and $I^{2}$ tests were used to assess the heterogeneity of clinical trial results and determine the analysis model (fixed-effects model or random-effects model). When the chi-squared test $P$ value was $\leq 0.05$ and $I^{2}$ test value was $>50 \%$, it was defined as high heterogeneity and assessed by random-effects model. When the 
chi-squared test $P$ value was $>0.05$ and $I^{2}$ tests value was $\leq 50 \%$, it was defined as acceptable heterogeneity data and assessed by fixed-effects model. For further assessment of heterogeneity, diagnostic threshold analysis was performed based on the correlation (Spearman's) between the logit of sensitivity and the logit of [1-specificity]. When a threshold effect occurs, the sensitivity and specificity of the investigated study exhibits negative correlation (or a positive correlation between sensitivity and [1-specificity]). Therefore, a strong positive correlation between sensitivity and [1-specificity] suggests the presence of a threshold effect. When heterogeneity caused by threshold effect was observed, a summary receiver operating characteristic (SROC) curve was plotted. This method was appropriate given that the global sensitivity and specificity values were overestimated. In such cases, analysis of the ROC panel points, as well as analysis of the SROC curve, was recommended. Deeks' Funnel Asymmetry Plot was used to identify the publication bias.

\section{Results}

\section{Characteristics of included studies}

A total of 2092 articles were searched by the indexes. After screening the titles and abstracts, 1986 articles were excluded, leaving 106 articles for further selection. During full-text screening, 63 articles were excluded due to the following criteria: unqualified outcomes [7], theoretical research or review [8], and has non clinical outcome [9]. At last, 43 studies [7-50] with 3133 hips were involved in the final meta-analysis. The selection process was presented in Fig. 1. The main characteristics of the included studies were summarized in Table 1 . The basic information included number of hips, age, and gender.

\section{Diagnostic accuracy}

All the included studies reported the results of the accuracy of MRI of early osteonecrosis of the femoral head. Based on the correlation (Spearman's $R=-0.209$, $P=0.589)$ between the logit of sensitivity and the logit of [1-specificity], there was no threshold effect.

Based on the chi-squared test $(Q=166.45, P=0.000)$ and $I^{2}$ tests $\left(I^{2}=74.6 \%\right)$, heterogeneity was high, so we chose the random-effects model to analyze the sensitivity. The global sensitivity was $93.0 \%$ (95\% CI 92.0 $94.0 \%$, Fig. 2). Based on the chi-squared test $(Q=144.43$, $P=0.000)$ and $I^{2}$ tests $\left(I^{2}=70.9 \%\right)$, heterogeneity was high. Therefore, we chose the random-effects model to analyze the specificity, and the global specificity was 91.0\% (95\%CI 89.0-93.0\%, Fig. 3).

Based on the chi-squared test $(Q=125.33, P=0.000)$ and $I^{2}$ tests $\left(I^{2}=66.5 \%\right)$, heterogeneity was high, so we
Table 1 The basic characteristics description of included studies

\begin{tabular}{|c|c|c|c|}
\hline Study & No. of hip & Gender & Age \\
\hline Genez BM 1998 & 11 & $4 \mathrm{M}, 3 \mathrm{~F}$ & $11-35$ \\
\hline Robinson HJ 1989 & 96 & - & - \\
\hline Hauzeur JP 1989 & 49 & - & - \\
\hline Zhang X 1994 & 30 & $26 \mathrm{M}$ & $24-58$ \\
\hline Ryu JS 2002 & 32 & $14 \mathrm{M}, 10 \mathrm{~F}$ & 39.5 \\
\hline Liu Jihua 2004 & 72 & $40 \mathrm{M}, 8 \mathrm{~F}$ & 43.6 \\
\hline Chen Lei 2005 & 62 & $3 \mathrm{M}, 21 \mathrm{~F}$ & 31.8 \\
\hline Zhou Hongmei 2006 & 91 & $23 \mathrm{M}, 18 \mathrm{~F}$ & $30-60$ \\
\hline Xie Zhongwei 2010 & 68 & $34 \mathrm{M}, 16 \mathrm{~F}$ & 34 \\
\hline Sun Lili 2015 & 50 & $36 \mathrm{M}, 14 \mathrm{~F}$ & 50.1 \\
\hline Fang Wu 2017 & 35 & $20 \mathrm{M}, 15 \mathrm{~F}$ & 57.1 \\
\hline Feng Zhanyou 2017 & 71 & $33 \mathrm{M}, 38 \mathrm{~F}$ & 61.5 \\
\hline Cheng Houpei 2016 & 65 & $40 \mathrm{M}, 25 \mathrm{~F}$ & 41.14 \\
\hline Qiu Pengdong 2012 & 59 & $31 \mathrm{M}, 23 \mathrm{~F}$ & 38.5 \\
\hline Zheng Liwen 2013 & 98 & $35 \mathrm{~F}, 21 \mathrm{M}$ & 38.2 \\
\hline Cui Baoli 2014 & 114 & - & 32.3 \\
\hline Xie Yan 2014 & 81 & $37 \mathrm{M}, 32 \mathrm{~F}$ & $31-62$ \\
\hline Jia Hong 2017 & 40 & $25 \mathrm{M}, 15 \mathrm{~F}$ & 56.6 \\
\hline Zhang Kaixiang 2016 & 100 & $62 \mathrm{M}, 38 \mathrm{~F}$ & 42.3 \\
\hline Lin Chen 2017 & 42 & - & 52.87 \\
\hline Lu Chun 2016 & 52 & $28 \mathrm{M}, 24 \mathrm{~F}$ & 51.2 \\
\hline Ding Qinmei 2011 & 62 & $31 \mathrm{M}, 15 \mathrm{~F}$ & 41.2 \\
\hline Chen Longhua 2015 & 196 & $75 \mathrm{M}, 45 \mathrm{~F}$ & 58.4 \\
\hline Luo Zian 2017 & 93 & $39 \mathrm{M}, 21 \mathrm{~F}$ & 32.3 \\
\hline Tan Zhihong 2016 & 131 & $54 \mathrm{M}, 32 \mathrm{~F}$ & 58.68 \\
\hline Wang Yuli 2017 & 50 & $38 \mathrm{M}, 12 \mathrm{~F}$ & 53.8 \\
\hline Wang Wenbin 2012 & 72 & $29 \mathrm{M}, 11 \mathrm{~F}$ & 52.5 \\
\hline Liu Xianzhi 2017 & 29 & $16 \mathrm{M}, 13 \mathrm{~F}$ & 52.26 \\
\hline Liu Feng 2017 & 43 & $25 \mathrm{M}, 18 \mathrm{~F}$ & 58.14 \\
\hline Guo Hongbin 2017 & 70 & $39 \mathrm{M}, 31 \mathrm{~F}$ & 44.86 \\
\hline Lin Yi 2015 & 90 & $30 \mathrm{M}, 20 \mathrm{~F}$ & 45.6 \\
\hline Wang Linhong 2014 & 183 & $60 \mathrm{M}, 40 \mathrm{~F}$ & 47 \\
\hline Fang Chaohui 2014 & 122 & $56 \mathrm{M}, 38 \mathrm{~F}$ & 64.8 \\
\hline Li Yan 2014 & 98 & 69 M, $29 \mathrm{~F}$ & 51.52 \\
\hline Liu Dailiang 2015 & 86 & $23 \mathrm{M}, 20 \mathrm{~F}$ & 55.36 \\
\hline Xiang Zhenghua 2014 & 61 & $35 \mathrm{M}, 26 \mathrm{~F}$ & 52.8 \\
\hline Wu Shiping 2017 & 75 & $51 \mathrm{M}, 24 \mathrm{~F}$ & 40.7 \\
\hline Wang Kun 2017 & 56 & $34 \mathrm{M}, 22 \mathrm{~F}$ & 38.74 \\
\hline Cai Huaiwei 2017 & 69 & $45 \mathrm{M}, 24 \mathrm{~F}$ & 54.6 \\
\hline Li Yanming 2015 & 37 & - & 52.6 \\
\hline Ji Xuewen 2017 & 50 & $28 \mathrm{M}, 22 \mathrm{~F}$ & 48.3 \\
\hline Wang Sihe 2013 & 86 & $32 \mathrm{M}, 27 \mathrm{~F}$ & 32.4 \\
\hline Shen Wen 2014 & 56 & $21 \mathrm{M}, 15 \mathrm{~F}$ & 45.3 \\
\hline
\end{tabular}




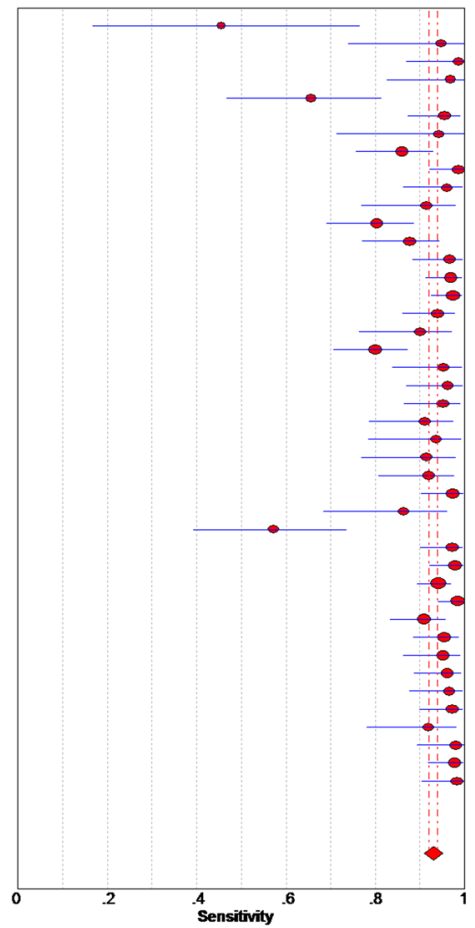

Genez BM 1998 Robinson HJ 1989 Haureur $J P 1989$ Ryu JS 2002 Cin Jhuna 2004 nou Hongmei2006 Sun Li 2015 Fang Wu 2017 Cheng Houpei 2016

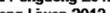
Gie Bact 2014 de Yan 2014 Jia Hong 2017 Zhang Kaixiang 2016 (4) Ding Oinmei 2011 Chen Ionghat 2015 Tan Zithong 2016 Wang Yut 2017 in Xianti 2017 Guo Hongbin 2017 Wh reolis 2014 i Yan2014 ualiang 2015 Xang Zhenghua 2014 Wu Shiping 2017 (1) I Yarming 2015 Ji Xuewen 2017 Wang She 2013
Shen Wen 2014

Sensitivity $(95 \% \mathrm{cn}$

$0.45 \quad(0.17-0.77)$

$0.95 \quad(0.74-1.00)$
0.99
0.97

$0.97 \quad(0.83-1.00)$

$0.66 \quad(0.47-0.81)$

$\begin{array}{ll}0.95 & (0.87-0.99) \\ 0.94 & (0.71-1.00)\end{array}$

$\begin{array}{ll}0.94 & (0.71-1.00) \\ 0.86 & (0.76-0.93)\end{array}$

$\begin{array}{ll}0.86 & (0.76-0.93) \\ 0.99 & (0.92-1.00)\end{array}$

$0.80 \quad(0.69-0.89)$

$0.88 \quad(0.77-0.95)$

$\begin{array}{ll}0.97 & (0.88-1.00) \\ 0.97 & (0.91-0.99)\end{array}$

$0.97(0.93-0.99)$

$0.94 \quad(0.86-0.98)$

$\begin{array}{ll}0.90 & (0.76-0.97) \\ 0.80 & (0.71-0.87)\end{array}$

$\begin{array}{ll}0.80 & (0.71-0.87) \\ 0.95 & (0.84-0.99)\end{array}$

$\begin{array}{ll}0.95 & (0.84-0.99) \\ 0.96 & (0.87-1.00\end{array}$

$\begin{array}{ll}0.95 & (0.87-0.99) \\ 0.91 & (0.79-0.98\end{array}$

$0.94 \quad(0.79-0.99)$

$0.91 \quad(0.77-0.98)$

$0.92 \quad(0.81-0.98)$

$\begin{array}{ll}0.97 & (0.90-1.00 \\ 0.86 & 0.68-0.96\end{array}$

$0.57 \quad 0.39-0.74$

$0.97 \quad(0.90-1.00)$

$0.98 \quad(0.92-1.00)$

$0.98 \quad(0.94-1.00)$

$0.91 \quad(0.83-0.96)$
$0.95 \quad(0.89-0.99)$

$0.95 \quad(0.86-0.99)$

$0.96(0.89-0.99)$

$\begin{array}{ll}0.96 & (0.88-1.00) \\ 0.97 & (0.90-1.00)\end{array}$

$0.92 \quad(0.78-0.98)$

$0.98 \quad(0.89-1.00)$

$\begin{array}{ll}0.98 & (0.92-1.00) \\ 0.98-1.00)\end{array}$

Pooled Sensitivity $=0.93$ (0.92 to 0.94$)$ $\begin{aligned} \text { Chi-square }=165.45 ; & \text { df }=42(p=0.0000) \\ \text { nconsistency }(1-\text {-qquare }) & =74.6 \%\end{aligned}$

Fig. 2 Forest plot showing the sensitivity values of MRI of early osteonecrosis of the femoral head

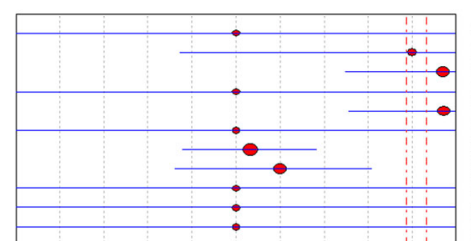

Genez BM 1998

Robinson HJ 1989

Haurzeur JP 1989

Zhang X 1994

Liv Jhua 2004

Zhou Hongmei2006

Xie Zhongwei 2010

Sun Lا 2015

Fang Wu 2017

Cheng Houpei 2016

Oin Pengdong 201

Xie Yan 2014

Jia Hong 2017

Zhang Kaixiang 2016

in Chen 2017

Lu Chin 2016

Chen Longhua 2015

Luo Żan 2017

Tan Zhihong 2016

Wang Wenbin 2012

Lin Xiandin 2017

Liv Feng 2017

Guo Hongbin 2017

in Y2015

Wang Linhong 2014

Li Yan2014

Liu Dariang 2015

Xiang Zhenghwa 2014

Wu Shiping 2017

Wang Kun 2017

L Y Yanming 2015

Ji Xuewen 2017

Wang Sihe 2013

Specificity ( $95 \%$ C)

$0.50 \quad(0.00-1.00)$

$0.90 \quad(0.37-1.00)$

$0.97 \quad(0.75-1.00)$

$0.50 \quad(0.00-1.00)$

$0.97 \quad(0.76-1.00)$

$0.53 \quad(0.38-0.68$

$0.60 \quad(0.36-0.81)$

$0.50 \quad(0.00-1.00)$

$0.50 \quad(0.00-1.00)$

$\begin{array}{ll}0.50 & (0.00-1.00) \\ 0.50 & (0.00-1.00)\end{array}$

$0.50 \quad(0.00-1.00)$

$0.50 \quad(0.00-1.00)$

$\begin{array}{ll}0.50 & (0.00-1.00) \\ 0.50 & (0.00-1.00)\end{array}$

$0.50 \quad(0.00-1.00)$

$0.50 \quad(0.00-1.00)$

$0.50 \quad(0.00-1.00)$

$0.50 \quad(0.00-1.00)$

$0.50 \quad(0.00-1.00)$

$0.50 \quad(0.00-1.00)$

$\begin{array}{ll}0.99 & (0.95-1.00) \\ 0.98 & (0.92-1.00)\end{array}$

$\begin{array}{ll}0.98 & (0.92-1.00) \\ 0.98 & (0.95-1.00)\end{array}$

0.98
0.50
$(0.00-1.00)$

$0.50 \quad(0.00-1.00)$

$0.50 \quad(0.00-1.00)$

$0.98 \quad(0.93-1.00)$

$\begin{array}{ll}0.50 & (0.00-1.00) \\ 0.50 & (0.00-1.00)\end{array}$

$0.50 \quad(0.00-1.00)$

$0.50 \quad(0.00-1.00)$

$0.50 \quad(0.00-1.00)$

$0.50 \quad(0.00-1.00)$

$0.50 \quad(0.00-1.00)$

$.50 \quad(0.00-1.00)$

$0.50 \quad(0.00-1.00)$

$0.50 \quad(0.00-1.00)$

$0.50 \quad(0.00-1.00)$

$\begin{array}{ll}0.50 & (0.00-1.00) \\ 0.50 & (0.00-1.00)\end{array}$

Poled Speciticity $=0.91$ ( 0.89 to 0.93 )

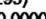

Chi-square $=144.43 ; \mathrm{df}=42(\mathrm{p}=0.0000)$

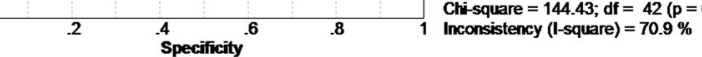

Fig. 3 Forest plot showing the specificity values of MRI of early osteonecrosis of the femoral head 


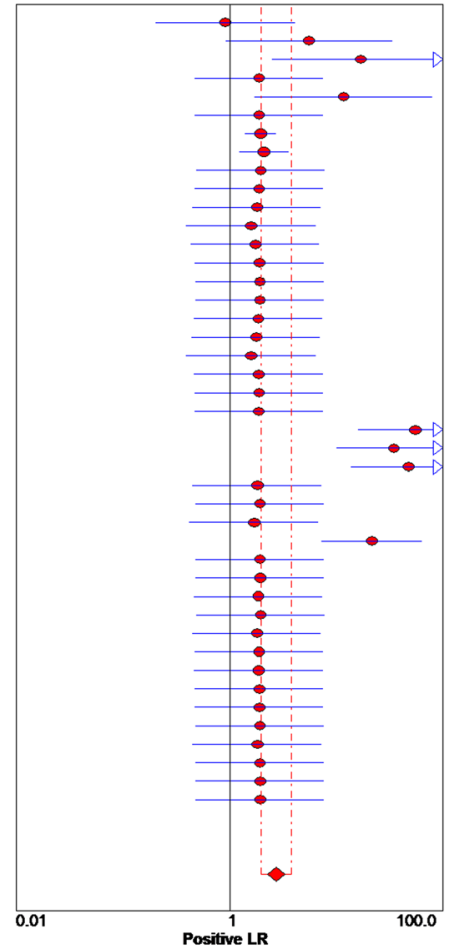

Genez BM 1998 Robinson HJ 1989 Hauzeur JP 1989 Zhang X 1994
Ryu JS 2002 Lin Jhur 2004 Thou Hongmei2006 Xie Zhongwei 2010 Sun L 2015 Fang Wu 2017 Cheng Houpei 2016 ain Pengdong 2012 Theng Liwen 2013 Cui Baol 2014

Xie Yan 2014

Zhang Kaixiang 2016 Lin Chen 2017
Lu Chin 2016 Ding Oinmei 2011 Luo Zan 2017 Tan Zhihong 2016 Wang Wenbin 2012 Lin Xianzzit 2017 Guo Hongbin 2017 in YR2015 Wang Linhong 2014 Fang Chaohui Liu Dariang 2015 Xiang Zhenghwa 2014 Wang Kun 2017 Cai Huaiwei 2017 Li Yanming 2015 Wang She 2013 Random Effects Model
Pooled Positive $L R=2.74$ (1.98 to 3.79 ) Cochran- $Q=125.33 ; \mathrm{df}=42(\mathrm{p}=0.0000)$ Inconsistency (I-square) $=66.5 \%$

Fig. 4 Forest plot showing the positive likelihood ratio of MRI

Positive LR (95\% Cl)

$0.92 \quad(0.20-4.18)$

$\begin{array}{ll}5.55 & (0.92-33.36) \\ 16.99 & (2.53-114.07)\end{array}$

$1.90(0.47-7.63)$

$11.73(1.72-80.07)$

$1.90 \quad(0.47-7.59)$

$\begin{array}{ll}1.96 & (1.40-2.75) \\ 2.11 & (1.25-3.58)\end{array}$

$1.96 \quad(0.49-7.83)$

$\begin{array}{ll}1.90 & (0.48-7.62) \\ 1.81 & (0.45-7.25)\end{array}$

$1.74 \quad(0.43-6.99)$

$1.92(0.48-7.67)$

$1.93(0.48-7.72)$

$1.94 \quad(0.48-7.76)$

$\begin{array}{ll}1.87 & (0.47-7.47 \\ 1.78 & (0.44-7.15)\end{array}$

$\begin{array}{ll}1.78 & (0.44-7.15) \\ 1.59 & (0.40-6.40)\end{array}$

$1.88 \quad(0.47-7.55)$

$1.91(0.48-7.63)$

$1.89 \quad(0.47-7.56)$

$54.85 \quad(15.98-188.23)$

$47.67 \quad(13.16-118.25)$

$1.82(0.45-7.31)$

$1.93 \quad(0.48-7.73)$

$1.70 \quad(0.42-6.85)$
$21.48 \quad(7.35-62.74)$

$1.93 \quad(0.48-7.72)$

$1.95 \quad(0.49-7.78)$

$\begin{array}{ll}1.88 & (0.47-7.50 \\ 1.96 & (0.49-7.84\end{array}$

$1.81 \quad(0.45-7.24)$

$1.90 \quad(0.47-7.59)$

$1.89 \quad(0.47-7.56)$

$1.91 \quad(0.48-7.64)$

$\begin{array}{ll}1.91 & (0.48-7.65) \\ 1.93 & (0.48-7.72)\end{array}$

$1.93(0.48-7.72)$

$1.82(0.45-7.29)$

$\begin{array}{ll}1.94 & (0.49-7.7) \\ 1.95 & (0.49-7.79)\end{array}$

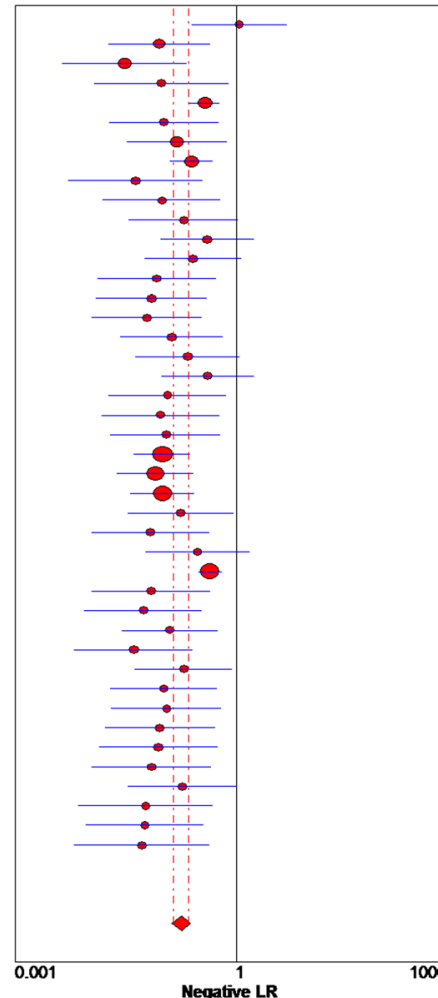
Robinson HJ 1989 Hauzeur JP 1989 Ryu JS 2002 Liv Jhura 2004

Chen Lei 2005

Zhou Hongmei2006

Xie Zhongwei 2010

Sun L面 2015

Fang Wu 2017

Cheng Houpei 2016

Qiu Pengdong 2012

Zheng Liwen 2013

$X$ Xie Yan 2014

Jia Hong 2017

Thang Kaixiang 2016

in Chen 2017

Lu Chun 2016

Ding Oinmei 2011

Luo Zan 2017

Tan Zhinong 2016

Wang Yus 2017

in Xianzhi 2017

iu Feng 2017

Guo Hongbin 2017

in Yi2015

Wang Linhong 2014

$L Y$ Yan2014

iv Dariang 2015

Xiang Zhenghua 2014

Wu Shiping 2017

Wang Kum 2017

Li Yanming 2015

Ji Xuewen 2017

Wang She 2013
Shen Wen 2014

Negative LR (95\% cn

$1.08 \quad(0.25-4.76)$

$0.09 \quad(0.02-0.44)$

$0.10 \quad(0.01-0.78)$

$0.37(0.23-0.60)$

$0.10(0.02-0.58)$

$0.16 \quad(0.03-0.74)$

$0.25 \quad(0.13-0.47)$

$0.04(0.01-0.36)$

$0.10 \quad(0.02-0.62)$

$0.19 \quad(0.04-1.07)$

$0.26 \quad(0.06-1.18)$

$0.07 \quad(0.01-0.40)$

$0.06(0.01-0.34)$

$0.13(0.03-0.67)$

0.22 (0.04-1.13)

$0.41 \quad(0.10-1.71)$

$0.12 \quad(0.02-0.73)$

$0.09 \quad(0.01-0.59)$

$0.11 \quad(0.02-0.62)$

$0.10 \quad(0.04-0.24)$

$0.10 \quad(0.04-0.27)$

$\begin{array}{ll}0.10 & (0.04-0.27) \\ 0.18 & (0.03-0.91)\end{array}$

$0.07 \quad(0.01-0.43)$

$0.30 \quad(0.06-1.53)$

$0.44(0.30-0.64)$

$0.07(0.01-0.45)$

$0.05 \quad(0.01-0.35)$

$0.13 \quad(0.03-0.56)$

$0.04(0.01-0.26)$

$0.19 \quad(0.04-0.87)$

$\begin{array}{ll}0.10 & (0.02-0.54) \\ 0.11 & (0.02-0.63)\end{array}$

$\begin{array}{ll}0.11 & (0.02-0.63) \\ 0.09 & (0.02-0.52)\end{array}$

$\begin{array}{ll}0.09 & (0.02-0.52) \\ 0.09 & (0.01-0.55)\end{array}$

$0.07(0.01-0.45)$

$0.18 \quad(0.03-1.02)$

$0.06 \quad(0.01-0.48)$

$\begin{array}{ll}0.06 & (0.01-0.36) \\ 0.05 & (0.01-0.43)\end{array}$

Fixed Effects Model

Pooled Negative $L R=0.18$ (0.14 to 0.23 )

Cochran- $Q=69.58 ;$ df $=42(p=0.0047)$

Negative LR

1000.0 Inconsistency (I-square) $=39.6 \%$

Fig. 5 Forest plot showing the negative likelihood ratio of MRI 
chose random-effects model to analyze the positive likelihood ratio, and the global positive likelihood ratio was 2.74 (95\% CI 1.98-3.79, Fig. 4). Therefore, a positive MRI result was increased by 2.74 -fold in the odds of an accurate diagnosis of patients who actually had early osteonecrosis of the femoral head. Based on the chi-squared test $(Q=69.58, P=0.005)$ and $I^{2}$ tests $\left(I^{2}=\right.$ $39.6 \%$ ), with low heterogeneity, we chose the fixed-effects model to analyze the negative likelihood ration. The global negative likelihood ratio was 0.18 (95\% CI $0.14-0.23$, Fig. 5), indicating the use of MRI, which was close to zero. Specifically, the odds of a false-positive result were increased by only a factor of 0.18 .

Based on the chi-squared test $(Q=59.71, P=0.037)$ and $I^{2}$ tests $\left(I^{2}=29.7 \%\right)$, heterogeneity was low, so we chose the fixed-effects model to analyze the DOR, with the global DOR being 27.27 (95\% CI 17.02-43.67, Fig. 6). And the odds of a positive MRI result were 27.27-fold higher among individuals with early osteonecrosis of the femoral head compared to those without the disease. The area under the SROC was 93.38\% (AUC $=93.38 \%$; 95\% CI 90.87\%-95.89\%, Fig. 7), indicating high accuracy.

\section{Conclusions}

Several systematic reviews and meta-analysis have been published concerning the diagnostic accuracy of MRI of early osteonecrosis of the femoral head. Li et al. [51] found that the sensitivity and specificity of MRI were 95\%(95\% CI 94-96\%) and 77\%(95\% CI 70-83\%), respectively. Moreover, the DOR was $31.89 \%(95 \%$ CI $17.32-$ $58.70 \%$ ), and the AUC under the SROC was 0.9166. MRI was associated with high diagnostic accuracy in the patients with suspected early ANFH. Song et al. [52], who included 21 articles, reported that MRI was more effective than CT in diagnosing ANFH. Significant statistical difference was identified between them (OR, 0.13; 95\% CI 0.03-0.51). Su et al. [53], who included 8 studies of 515 patients, found the ANFH positive rate between $\mathrm{CT}$ and MRI was statistically significant (OR, 0.12 ; $95 \%$ CI $0.04-0.33$ ), so as the early stage positive rate (OR, $0.45 ; 95 \%$ CI 0.26-0.78). Therefore, MRI appears to be a promising diagnostic tool for avascular necrosis of the femoral head.

However, there were several limitations in this analysis: (1) differences in the inclusion and exclusion criteria for

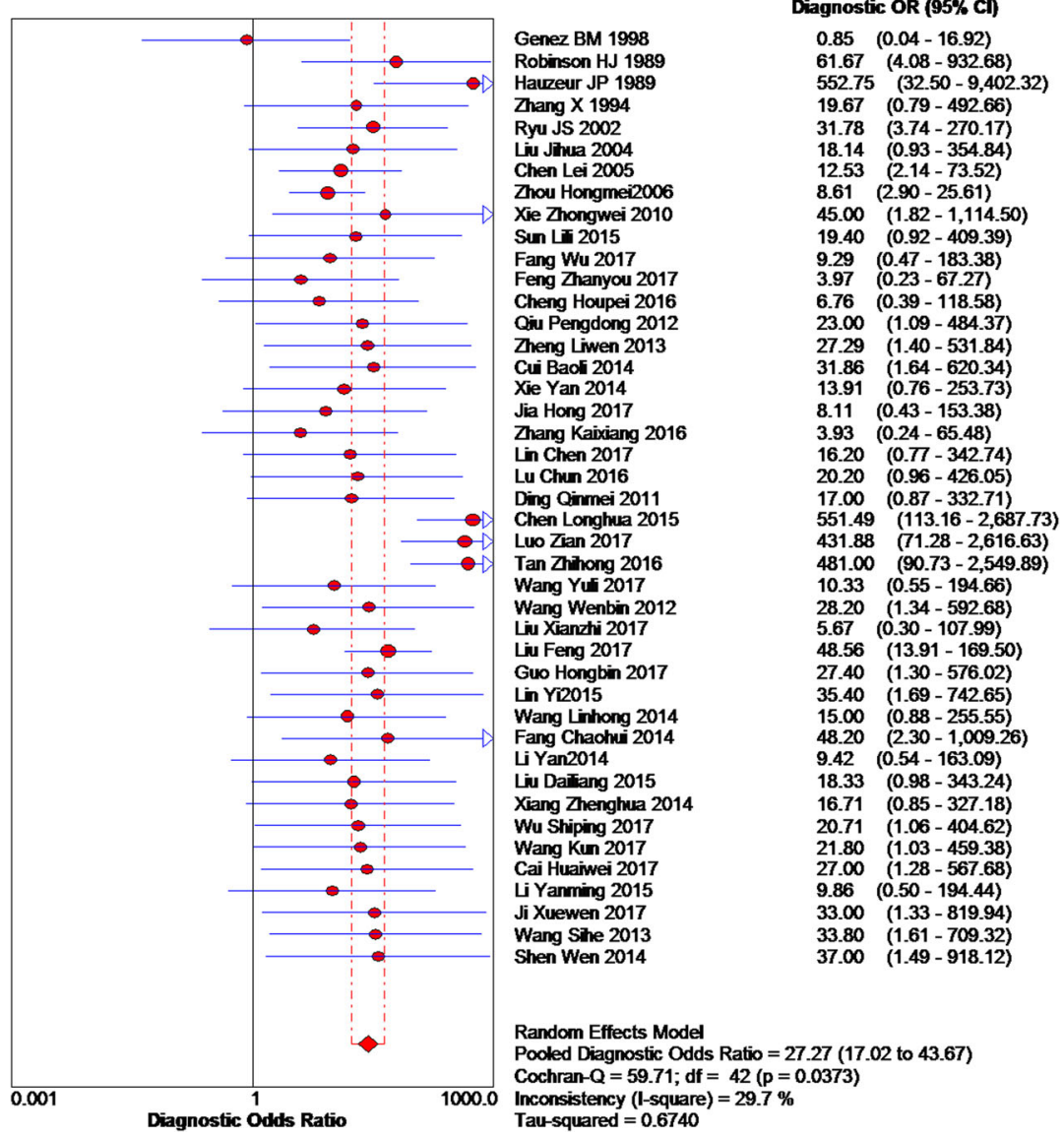

Fig. 6 Forest plot showing the diagnostic odds ratio of MRI of early osteonecrosis of the femoral head 


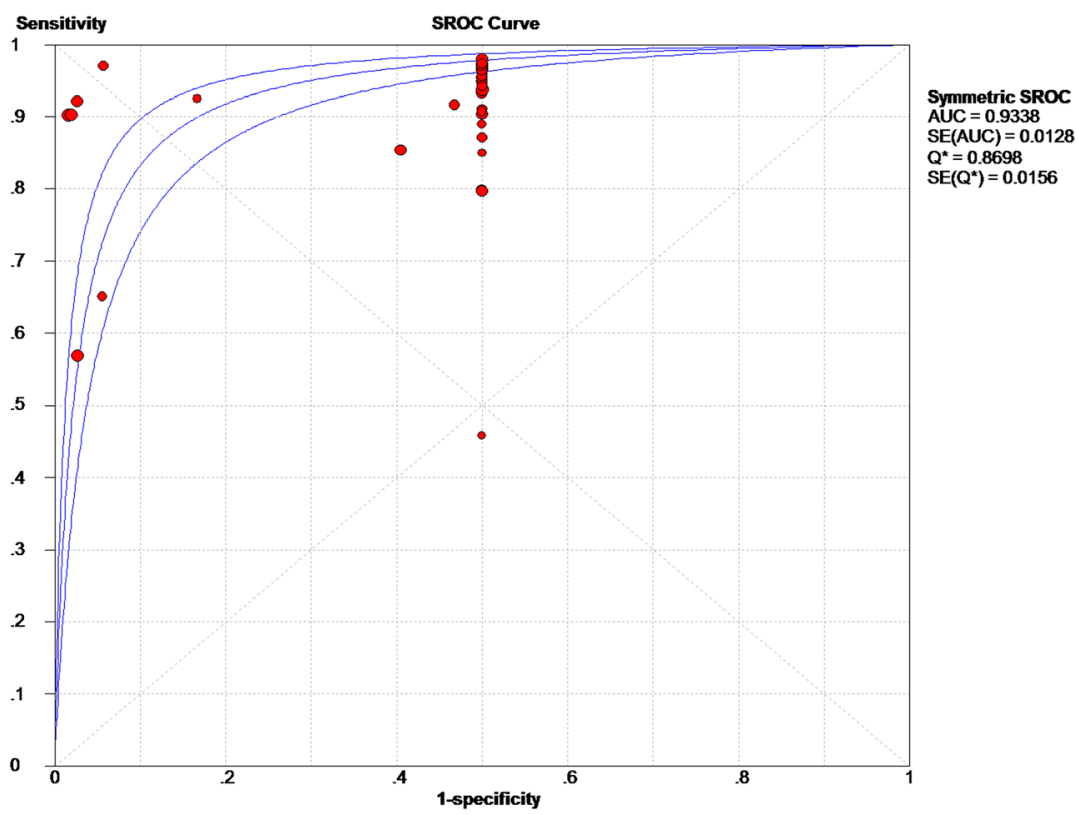

Fig. 7 Summary ROC plots for diagnostic accuracy of MRI of early osteonecrosis of the femoral head

patients, (2) different patients with previous disease and treatments were unavailable, (3) all the included studies were from English and Chinese articles, which may be the source of bias, (4) the fluency of technicians between different studies varied, and (5) pooled data were used for analysis, and individual patients' data were unavailable, which limited a more comprehensive analysis.

In summary, in this systematic review and meta-analysis, MRI as a diagnostic method is associated with higher accuracy for detecting ANFH. More studies and randomized controlled trails with high-quality and large samples are warranted for further evaluation.

\section{Abbreviations}

ANFH: Avascular Necrosis of Femur Head; FN: False negative; FP: False positive; MRI: Magnetic resonance imaging; SROC: Summary receiver operating characteristic; TN: True negative; TP: True positive

\section{Authors' contributions}

YZZ have made substantial contributions to the conception and design of the study. XYC and XCL searched the literature, extracted data from the collected literature, and analyzed the data. JC and WZ wrote the manuscript. YYZ and ZT revised the manuscript. All authors approved the final version of the manuscript.

Ethics approval and consent to participate

Not applicable.

\section{Competing interests}

The authors declare that they have no competing interests.

Received: 22 April 2018 Accepted: 15 May 2018

Published online: 04 July 2018

\section{References}

1. Mankin HJ. Nontraumatic necrosis of bone (osteonecrosis). N Engl J Med. 1992;326(22):1473-9
2. Castro FP Jr, Barrack RL. Core decompression and conservative treatment for avascular necrosis of the femoral head: a meta-analysis. Am J Orthop. 2000; 29(3):187-94.

3. Hong YC, Zhong HM, Lin T, Shi JB. Comparison of core decompression and conservative treatment for avascular necrosis of femoral head at early stage: a meta-analysis. Int J Clin Exp Med. 2015;8(4):5207-16.

4. Nakamura T, Matsumoto T, Nishino M, Tomita K, Kadoya M. Early magnetic resonance imaging and histologic findings in a model of femoral head necrosis. Clin Orthop Relat Res. 1997;334:68-72.

5. Ficat RP. Idiopathic bone necrosis of the femoral head. Early diagnosis and treatment. J Bone Joint Surg Br Vol. 1985;67(1):3-9.

6. Jawad MU, Haleem AA, Scully SP. In brief: Ficat classification: avascular necrosis of the femoral head. Clin Orthop Relat Res. 2012:470(9):2636-9.

7. Li YM. Comparative analysis of CT and MRI in diagnosis of femoral head necrosis. World Latest Med Inform. 2015:40:140.

8. Sun LL. Comparative study of CT and MRI in diagnosis of 100 cases of femoral head necrosis. China Health Stand Manag. 2015;10:29-30.

9. Wang YL. The value of contrast MRI and CT in the early diagnosis of femoral head necrosis. Chin J Trauma Disabil Med. 2017;25(21):47-8.

10. Zheng LW, Chen RH, Chen YH. The application value of $C T$ and MRI in early diagnosis of osteonecrosis of the femoral head. China Mod Doct. 2013; 51(11):90-1.

11. Cui BG. The application value of $C T$ and MRI in early diagnosis of osteonecrosis of the femoral head. Mod Med Imageol. 2014;23(4):366-9.

12. Xie $Y$. The value of $C T$ and $M R I$ in the diagnosis of early femoral head necrosis. Chin J Mod Drug Appl. 2014:8(2):70-1.

13. Jia $H$, Xiao YX, Zhang J, An L. The value of $C T$ and MRI in the diagnosis of femoral head necrosis. Gansu Med J. 2017:36(5):377-8.

14. Fang W, Yang WJ, Liang TH, Huang RS. Application value of $C T$ and MRI in the diagnosis of early femoral head necrosis. J Baotou Med Coll. 2017:33(1):55-6.

15. Feng ZY, Ma Z, Qu JX, Chen W. CT and magnetic resonance imaging (MRI) in the diagnosis of femoral head necrosis in comparison. Mod Diagn Treat. 2017;28(12):2293-5.

16. Zhang KX. MRI and CT diagnosis of avascular necrosis of the femoral head. World Clin Med. 2016;10(8):238.

17. Zi LL. Comparative analysis of CT and MRI in the diagnosis of early necrosis of femoral head. Mod Diagn Treat. 2015;(9):2093-4.

18. Qiu PD. Comparative analysis of $C T$ and MRI in the diagnosis of early necrosis of femoral head. Modern Medical Imageology. 2012;21(5):315-6. 
19. Lin C, Ren CP. Clinical value of MRI in the diagnosis of early necrosis of femoral head. J Med Imag. 2017;27(6):1203-5.

20. Sheng W. Comparative analysis of diagnostic value of $X$ - ray, $C T$ and $M R I$ in early diagnosis of avascular necrosis of femoral head. Mod Diagn Treat. 2014;2:352-3.

21. Lu C. Analysis on diagnosis value of MRI in early femoral head necrosis. Chin Foreign Med Res. 2016;14(19):56-7.

22. Ding QM. Comparison of X-ray, CT and MRI in the diagnosis of early avascular necrosis of femoral head. Chin J Primary Med Pharm. 2011;18(6):784-5.

23. Wang $\mathrm{SH}$. Application of spiral $\mathrm{CT}$ and high field MRI in the early diagnosis of adult femoral head necrosis. Jiangxi Med J. 2013;12:1288-9.

24. Chen LH. Clinical value of CT and MRI in diagnosis of early osteonecrosis of femoral head in adults. Mod Inst Med Treat. 2015;(2):8-10.

25. Luo ZA. Clinical value of CT and MRI in diagnosis of early osteonecrosis of femoral head in adults. China Med Device Inform. 2017;23(16):93-4.

26. Tan ZH, Wu Y, Zhang PM, Li XL. Clinical value of CT and MRI in diagnosis of early osteonecrosis of femoral head in adults. Mod Med Imageol. 2016;25(2):342-3

27. Ji XW. Patients with avascular necrosis of the femoral head using MRI, spiral CT examination contrast. China Reflexolocy. 2017;26(7):154-6.

28. Cai HW. Comparison of multislice spiral $C T$ and $M R I$ in the clinical diagnosis of femoral head necrosis. Int Med Health Guid News. 2017;23(5):731-3.

29. Wang WB. Comparative study of CT and MRI in patients with femoral head necrosis. Chin J CT MRI. 2012;10(6):102-3.

30. Liu XZ, Mu CL. Femoral head necrosis in patients with a comparative study of CT and mri diagnosis. J Imag Res Med Appl. 2017;1(7):7-8.

31. Liu F. Comparative analysis of CT and MRI radiological diagnosis of femoral head necrosis. Mod Diagn Treat. 2017;28(13):2476-7.

32. Guo HB. Comparative analysis of CT and MRI in diagnosis of avascular necrosis of femoral head. Pract Clin J Integ Tradit Chin West Med. 2017;17(8):98-9

33. Lin $Y$. Comparison of $C T$ and magnetic resonance imaging in diagnosis and treatment of femoral head necrosis. Guide China Med. 2015;9:149-50.

34. Wang LH. Effect of CT and MRI in diagnosing femoral head necrosis. Contemp Med. 2014;32:150-1.

35. Fang $\mathrm{CH}$. Diagnostic value of two methods of $\mathrm{MRI}$ and $\mathrm{CT}$ in avascular necrosis of femoral head. China Med Pharm. 2014;13:109-11.

36. Li Y. MRI combined with CT in the diagnosis of avascular necrosis of the femoral head. J Med Imaging. 2014;24(9):1596-8.

37. Zhang J, Peng WX, Y. YF. Observation on the MRI diagnosis on ischemic bone necrosis and surgical treatment. Chin Commun Doct. 2016;32(29):129-30.

38. Liu DL. The value of CT and MRI examination in the early diagnosis of adult femoral head necrosis. Med Inf. 2015;43:347.

39. Xiang ZH. Low field strength MRI diagnosis of avascular necrosis of femoral head. China Foreign Med Treat. 2014;22:188-9.

40. Wu SP. Diagnostic value of multislice spiral $C T$ and mill on femoral head necrosis. Henan Med Res. 2017;26(17):3164-5.

41. Wang K. Evaluation of the value of multislice spiral CT and MRI in the diagnosis of femoral head necrosis. Diet Health. 2017;4(19):323.

42. Xie ZW, Yuan YH. Early diagnosis of avascular necrosis of femoral head in adults. Chin J Lab Diagn. 2010;14(8):1317-9.

43. Genez BM, Wilson MR, Houk RW, Weiland FL, Unger HR Jr, Shields NN, et al. Early osteonecrosis of the femoral head: detection in high-risk patients with MR imaging. Radiology. 1988;168(2):521-4.

44. Robinson HJ Jr, Hartleben PD, Lund G, Schreiman J. Evaluation of magnetic resonance imaging in the diagnosis of osteonecrosis of the femoral head. Accuracy compared with radiographs, core biopsy, and intraosseous pressure measurements. J Bone Joint Surg Am. 1989;71(5):650-63.

45. Hauzeur JP, Pasteels JL, Schoutens A, Hinsenkamp M, Appelboom T, Chochrad I, et al. The diagnostic value of magnetic resonance imaging in non-traumatic osteonecrosis of the femoral head. J Bone Joint Surg Am. 1989;71(5):641-9.

46. Zhang X, Hu CM, Zhao GK, Sun L, Qin DM. The value of magnetic resonance imaging in the early diagnosis of noninvasive avascular necrosis of femoral head. Chin J Surg. 1994;32(9):523-5.

47. Ryu JS, Kim JS, Moon DH, Kim SM, Shin MJ, Chang JS, et al. Bone SPECT is more sensitive than MRI in the detection of early osteonecrosis of the femoral head after renal transplantation. J Nuclear Med. 2002;43(8):1006-11.

48. Liu JH, Zhang SP, Wang SH, Zuo SY. Comparative study of radionuclide scanning and MRI in diagnosis of avascular necrosis of femoral head in early adults. Acta Acad Med Qingdao Univ. 2006;42(4):285-8.
49. Chen L, Hong N, Du XK. Avascular necrosis in severe acute respiratory syndrome: MR imaging with radionuclide correlation. Chin J Med Imag Technol. 2005;21(2):298-300.

50. Zhou HM, Li RG, Cui B, Tang FM, Huang BL. Diagnostic value of CT and MRI in the early stage of adult femoral head necrosis. J Qiqihar Univ Med. 2006; 12(7):529-31.

51. Li YX, Jiang PQ. Meta-analysis of MRI diagnosis of early avascular necrosis of femoral head. Chin Foreign Med Res. 2013;30:143-6.

52. Song WT, Li Z, Li XM, Yang XF. Meta-analysis of CT and MRI diagnosis of avascular necrosis of femoral head. J Pract Radiol. 2010;26(2):221-6.

53. Su JJ, Wu GY, Zhu L, Liu GB. Meta-analysis of CT and MRI diagnosis of avascular necrosis of femoral head. J New Med. 2011;21(3):178-84.

\section{Ready to submit your research? Choose BMC and benefit from:}

- fast, convenient online submission

- thorough peer review by experienced researchers in your field

- rapid publication on acceptance

- support for research data, including large and complex data types

- gold Open Access which fosters wider collaboration and increased citations

- maximum visibility for your research: over $100 \mathrm{M}$ website views per year

At BMC, research is always in progress.

Learn more biomedcentral.com/submissions 